\title{
La publicidad sexista en España: eficacia de la Ley Orgánica de Medidas de Protección Integral contra la Violencia de Género ${ }^{1}$
}

\author{
Marián Navarro-Beltrá \\ marian.navarro@ua.es \\ Universidad de Alicante
}

Marta Martín Llaguno

marta.martin@ua.es

Universidad de Alicante

Recibido: 28-02-2012

Aceptado: 18-03-2012

\section{Resumen}

La Ley Orgánica de Medidas de Protección Integral contra la Violencia de Género se promulga en España en el año 2004. Esta normativa, entre otros asuntos, regula y penaliza la discriminación por sexo en la comunicación comercial. Con el objetivo de comprobar la eficacia de esta delimitación, se realiza un análisis de contenido de una muestra representativa de spots españoles presentados a "El Sol. El Festival Iberoamericano de la Comunicación Publicitaria" en 1999 y 2009. Los resultados muestran que no se producen grandes diferencias en el grado de sexismo mostrado en los spots antes y después de la creación de la ley considerada, sin embargo, sí se producen importantes disimilitudes por sexo.

Palabras clave: Violencia de género, publicidad, sexismo, análisis de contenido, legislación, estereotipos de género.

\begin{abstract}
The Organic Act on Integrated Protection Measures against Gender Violence was passed in Spain in 2004. This law, among other issues, regulates and penalizes gender-based discrimination in advertising. In order to observe the effectiveness of this delimitation, in this paper it is carried out a content analysis of a representative sample of Spanish advertisements presented to "El Sol. El Festival Iberoamericano de la Comunicación Publicitaria”, in 1999 and 2009. The results show that there are no major differences in the degree of sexism in the advertisements before and after this law was passed. However, gender-based substantial dissimilarities are produced.
\end{abstract}

Keywords: Gender-based violence, advertising, sexism, content analysis, legislation, gender stereotypes.

\footnotetext{
${ }^{1}$ La realización de este trabajo ha sido posible gracias a la beca FPI de la Generalitat Valenciana y a la colaboración de la AEACP que nos ha permitido acceder a las bases de datos de "El Sol. El Festival Iberoamericano de la Comunicación Publicitaria”.
} 


\section{Introducción. La publicidad sexista en la legislación española}

Con el propósito de eliminar las diferencias entre mujeres y hombres en las sociedades desarrolladas se han llevado a cabo diversas políticas públicas. Éstas se inician gracias al "reconocimiento de que el Estado o los poderes públicos pueden y deben hacer algo para paliar las desigualdades de género y la discriminación de la mujer" (Bustelo, 2001: 135).

En concreto, para erradicar la violencia ejercida contra la población femenina, los medios de comunicación han sido considerados por normativas públicas de diferentes países como instrumentos capaces de coadyuvar a fomentar este tipo de agresiones ${ }^{2}$. Esta situación se produce al considerar a los mass media como agentes de socialización (Dietz, 1998) y al estimar que los discursos mediáticos pueden dificultar la existencia de una relación equilibrada entre mujeres y hombres, así, este tipo de relación es esencial si se pretende erradicar algún día la violencia machista (Guarro, 2004).

En el caso concreto de España, en el año 2004 se promulga la Ley Orgánica de Medidas de Protección Integral contra la Violencia de Género que, de manera extensa, trata de prevenir estas agresiones a través de distintos ámbitos de aplicación.

De esta forma, tal y como afirma la propia Ley, "la conquista de la igualdad y el respeto a la dignidad humana y la libertad de las personas tienen que ser un objetivo prioritario en todos los niveles de socialización" (Ley Orgánica de Medidas de Protección Integral Contra la Violencia de Género, 2004: 42167). Por este motivo, presta especial atención a la educación, a los mass media y, concretamente, a la publicidad, pues ésta también es considerada como un importante agente de socialización (Spirek y Glascock, 1998) que puede conllevar una gran influencia en la población.

Con referencia a los manifiestos publicitarios, la entrada en vigor de esta normativa supone la modificación de la Ley General de Publicidad (1988) que, entre otras cuestiones, empieza a considerar que no es lícita aquella comunicación comercial "que atente contra la dignidad de la persona o vulnere los valores y derechos reconocidos en la constitución, especialmente a los que se refieren sus artículos 18 y 20, apartado 4 .

Se entenderán incluidos en la previsión anterior los anuncios que presenten a las mujeres de forma vejatoria, bien utilizando particular y directamente su cuerpo o partes del mismo como mero objeto desvinculado del producto que se pretende promocionar, bien su imagen asociada a comportamientos estereotipados que vulneren los fundamentos de nuestro ordenamiento coadyuvando a generar la violencia a que se refiere la Ley Orgánica de medidas de protección integral contra la violencia de género" (Ley Orgánica de Medidas de Protección Integral Contra la Violencia de Género, 2004: 42183).

\footnotetext{
${ }^{2}$ A modo únicamente de ejemplo: Argentina (2009): Ley 26.485 de Protección Integral Para Prevenir, Sancionar y Erradicar la Violencia Contra las Mujeres en los Ámbitos en que Desarrollen sus Relaciones Interpersonales. Ley Orgánica 1/2004, de 28 de diciembre, de Medidas de Protección Integral Contra la Violencia de Género. En Boletín Oficial del Estado, 29 de diciembre de 2004, nº 313, pp. 42166-42197; México (2007): "Ley General de Acceso a las Mujeres a una Vida Libre de Violencia".
} 
No obstante, esta definición de publicidad sexista ha sido ligeramente modificada por la creación de la Ley por la que se Modifica el Régimen Legal de la Competencia Desleal y de la Publicidad Para la Mejora de la Protección de los Consumidores y Usuarios (2009). Específicamente, esta normativa introduce el artículo 14 de la Constitución y añade la palabra "discriminatoria" junto al vocablo "vejatoria" 3 . De esta forma, dicha variación no conlleva cambio alguno en los dos supuestos básicos de publicidad ilícita relacionados con el género y creados por la Ley Orgánica de Medidas de Protección Integral Contra la Violencia de Género (2004) -uso del cuerpo de la mujer o partes de éste como simple objeto sin vinculación con el producto promocionado y la utilización de la imagen de la mujer vinculada a comportamientos estereotipados capaces de coadyuvar a generar violencia de género-.

Consideramos que la promulgación de la Ley Orgánica de Medidas de Protección Integral Contra la Violencia de Género es necesaria ya que, pese a la creciente función social de las mujeres en España, la violencia machista continúa presente.

Así, en los últimos años, el nivel de igualdad educativo, laboral y legal femenino ha experimentado un continuo ascenso. En efecto, en España, la universidad se ha feminizado, pues en ella estudian más mujeres que hombres (García, 2010). Además, la integración de la mujer en el mercado laboral ha sido creciente. Así, en 1980 la tasa de actividad femenina era de un $27,77 \%$, en 1990 de un $34,56 \%$, en 2000 de un $41,76 \%$ y en 2010 de un $52,60 \%{ }^{4}$ (Instituto Nacional de Estadística, n.d.). No en vano, en este último periodo se han sucedido diversos planes de igualdad de oportunidades entre mujeres y hombres que, junto con las leyes promulgadas recientemente (conciliación, dependencia e igualdad) ${ }^{5}$ han propiciado estos avances.

Pese a esta inserción de la población femenina en la esfera pública, la III Macroencuesta Sobre la Violencia Contra las Mujeres (Sigma Dos, 2006) establece que el 9,6\% de las mujeres residentes en España mayores de edad son maltratadas. Así, en el año 2010 fallecieron a manos de sus parejas o exparejas 73 féminas, cifra superada únicamente

\footnotetext{
${ }^{3}$ El artículo 3a de la Ley General de Publicidad queda redactado como sigue y, por tanto, considera ilícita: "La publicidad que atente contra la dignidad de la persona o vulnere los valores y derechos reconocidos en la Constitución, especialmente a los que se refieren sus artículos 14, 18 y 20, apartado 4.

Se entenderán incluidos en la previsión anterior los anuncios que presenten a las mujeres de forma vejatoria o discriminatoria, bien utilizando particular y directamente su cuerpo o partes del mismo como mero objeto desvinculado del producto que se pretende promocionar, bien su imagen asociada a comportamientos estereotipados que vulneren los fundamentos de nuestro ordenamiento coadyuvando a generar la violencia a que se refiere la Ley Orgánica 1/2004, de 28 de diciembre, de Medidas de Protección Integral contra la Violencia de Género" (Ley por la que se Modifica el Régimen Legal de la Competencia Desleal y de la Publicidad Para la Mejora de la Protección de los Consumidores y Usuarios, 2009: 112053).

${ }^{4}$ Datos referentes al cuarto trimestre de cada año.

${ }^{5}$ Estas normativas son: España (1999): "Ley 39/1999, de 5 de noviembre, Para Promover la Conciliación de la Vida Familiar y Laboral de las Personas Trabajadoras"; España (2006): "Ley 39/2006, de 14 de diciembre, de Promoción de la Autonomía Personal y Atención a las Personas en Situación de Dependencia; España (2007): "Ley Orgánica 3/2007, de 22 de marzo, Para la Igualdad Efectiva de Mujeres y Hombres".
} 
desde el año 1999 por el 2008 con 76 mujeres muertas por violencia de género (Instituto de la Mujer, n.d.).

Ante este escenario de agresiones machistas contra la población femenina y la delimitación realizada por la Ley Orgánica de Medidas de Protección Integral Contra la Violencia de Género a la publicidad sexista por considerarla un elemento capaz de contribuir en el fomento de la violencia de género, parece urgente comprobar si los manifiestos publicitarios muestran una imagen menos discriminatoria de la mujer tras la promulgación de la normativa señalada.

\section{Objetivos}

El objetivo general del presente estudio se basa en comprobar la eficacia de la Ley Orgánica de Medidas de Protección Integral contra la Violencia de Género con relación a la existencia de sexismo en los manifiestos publicitarios. En concreto, se pretende analizar:

a) La utilización del cuerpo de hombres y mujeres y su vinculación con los productos anunciados;

b) La presentación estereotípica de ambos sexos y;

c) Los sesgos de género (visibilidad, paridad y empoderamiento) de mujeres y hombres).

\section{Método}

\subsection{Metodología}

El análisis de contenido ha sido la técnica escogida para cumplir los objetivos planteados, ya que se trata de un método utilizado frecuentemente por las investigaciones que analizan las representaciones de género en la publicidad (Kervin, 1990). Se han seleccionado los manifiestos publicitarios emitidos por televisión y cine al considerar "que la televisión, el cine y la publicidad se están convirtiendo, con el permiso de la familia, en los principales protagonistas en la construcción de los nuevos modelos sociales de convivencia" (González, 2007: 415).

\subsection{Población y muestra}

El universo de estudio ha estado conformado por los anuncios españoles presentados a la categoría "TV/Cine" del certamen "El Sol, el Festival Iberoamericano de la Comunicación 
Publicitaria"6 en las ediciones de 1999 y 2009 (cinco años antes y cinco años después de la promulgación de la Ley Orgánica de Medidas de Protección Integral contra la Violencia de Género). España inscribió en 1999 un total de 459 manifiestos publicitarios y en 2009 esta cifra se redujo a 242. Con el propósito de obtener el número específico de anuncios a analizar se ha recurrido al programa informático EPIDAT 3.1.

En concreto, el tamaño de la población ha sido obtenido para estimar proporciones, el nivel de precisión considerado ha sido del 10\% y el nivel de confianza establecido del $95 \%$, con un margen de error del $5 \%, \mathrm{P}=0,5^{7}$. De esta forma, se han analizado 80 spots inscritos en la edición de 1999 y 69 referentes al certamen realizado en 2009.

Los anuncios concretos a investigar también han sido seleccionados con EPIDAT 3.1. Así se han realizado dos muestreos probabilísticos aleatorios simples sin reposición con probabilidades iguales, uno para el año 1999 y otro para el 2009.

\subsection{Instrumentos de recogida de información}

Para la realización del presente trabajo se ha realizado un protocolo de codificación con 48 variables agrupadas en 4 grandes categorías: a) características del anuncio $^{8}$; b) características de los personajes principales; c) características de los personajes secundarios ${ }^{9}$ y; d) $\operatorname{Otros}^{10}$.

\subsection{Fiabilidad y validez}

Para conseguir un grado de fiabilidad intercodificadores aceptable, Wimmer y Dominick (1996: 184-185) recomiendan definir al máximo los límites de las categorías, adiestrar a los codificadores y realizar un ensayo previo. De esta forma, para el proceso de

\footnotetext{
${ }^{6}$ Este concurso se realiza, con una periodicidad anual, desde el año 1986. Hasta el 2002 la participación quedaba limitada a aquellas empresas cuya sede social estaba radicada en España. A partir de 2003 se permite la intervención de todas las naciones de habla portuguesa e hispana, de forma que en este festival se da cita la mejor creatividad iberoamericana y española, que por el reglamento del certamen, ha debido ser exhibida obligatoriamente. Para más información véase: http://www.elsolfestival.com/quees.asp [20/02/2012]; http://www.elsolfestival.com/reglamentoComun.asp?ord=1 [20/02/2012].

${ }_{7}$ En el caso de las proporciones y de no disponer de una estimación previa, se puede recurrir a la proporción $0,5(50 \%)$ por ser la que requiere un mayor tamaño de muestra (Marrugat, Vila, Pavesi, \& Sanz, 1998, p. 269).

${ }^{8}$ Número de ítem, año del festival, institución que inscribe el manifiesto publicitario, anunciante, agencia, categoría de productos, premio, medio, título, slogan, claim, público objetivo y duración.

${ }^{9}$ Tanto para los personajes protagonistas como para los secundarios se han analizado cuestiones como edad, sexo, perfil socioeconómico, profesiones, localización, relaciones, conductas sociales, estereotipos masculinos, estereotipos femeninos, cuerpo desvinculado del producto, vestuario, grado de insinuación, complexión física, argumentos de persuasión, aparición de personajes o voces por sexo, acción, dicción, recepción de acciones de los personajes y capacidad de tomar o acatar decisiones.

${ }^{10}$ Lenguaje sexista, recompensas para personajes masculinos, recompensas para personajes femeninos y sexo sobre el que recae la credibilidad.
} 
recogida de información se ha elaborado un protocolo de codificación y éste ha sido acompañado de un manual de instrucciones.

A su vez, se han realizado diversas pruebas en distintas sesiones con el objetivo de que los investigadores pusieran en práctica estos documentos y pudieran aprender a utilizarlo correctamente. En el caso de existir discrepancias en la codificación de las variables, los implicados en el proceso de codificación llegaron al consenso a través de la discusión.

Ambos investigadores, con anterioridad a realizar la codificación y con el propósito de conocer la fiabilidad intercodificadores, han analizado de forma independiente una misma submuestra $-20 \%$ del total de spots a codificar, es decir, 30 anuncios- y han obtenido un índice medio de Kappa de Cohen del 0,89 ${ }^{11}$.

Para conocer la fiabilidad intracodificadores se recurrió a la misma submuestra y al mismo índice. Así, dos meses después de la realización del proceso de codificación los investigadores volvieron a analizar los 30 spots anteriores. En concreto, uno de los analistas obtuvo un índice medio de Kappa de 0,93 y el otro de 0,88 .

Por su parte, al tener en cuenta la validez cabe destacar que las variables empleadas en el protocolo de codificación son el resultado de adaptar anteriores investigaciones. De esta forma, los ítems examinados proceden en parte del estudio realizado por Ruiz et al. (2004) que analiza la existencia de discriminación por género en las noticias de salud.

De este estudio se ha utilizado el concepto de sesgo de género, que ha sido opeacionalizado a través de la visibilidad (presencia equilibrada de hombres y mujeres), la paridad (representación igualitaria de ambos sexos diciendo, haciendo y padeciendo acciones) y el empoderamiento (muestra similar de varones y féminas tomando decisiones o acatando órdenes). Pese a que este indicador ha sido adaptado de las ciencias médicas ha demostrado su utilidad en la valoración del sexismo publicitario (Navarro-Beltrá y Martín-Llaguno, 2010).

Los roles de género considerados proceden en parte de los utilizados por MartínLlaguno, Quiles y López (2004), que analizan los manifiestos publicitarios de tabaco, y por García del Castillo, López-Sánchez, Quiles y García del Castillo-López (2009), que se centran en la publicidad de alcohol. En concreto, de estos estudios se utiliza la representación de personas como seductoras e independientes y se transforma el rol de objeto de deseo por objeto sexual.

Por su parte, del trabajo realizado por García (2003) se ha adaptado el grado de desnudez (se emplea la categoría de personajes vestidos y se aúnan en no vestidos totalmente los valores referentes a personas desnudas, en ropa interior, en ropa de baño y parcialmente desnudas), la localización (los escenarios propuestos se reducen a la esfera privada y a la esfera pública), las profesiones (se utilizan las categorías de actividad laboral tradicionalmente femenina, actividad laboral tradicionalmente masculina y actividad laboral neutra) y los argumentos (las premisas objetivas y las de opinión son transformadas en racionales y emocionales).

\footnotetext{
${ }^{11}$ Se ha calculado para cada variable que implicara un juicio por parte del investigador.
} 
La utilización del cuerpo desvinculado del producto promocionado procede directamente de la definición legal de publicidad sexista.

\subsection{Procedimiento}

Tras diseñar los instrumentos necesarios para recoger la información y obtener la muestra, para lo que ha sido necesario acceder a la base de datos de "El Sol", se ha realizado la codificación de los spots seleccionados a través del programa informático SPSS versión 15. Finalmente, para analizar los datos se han agrupado los personajes protagonistas y los secundarios y se ha recurrido a las tablas de respuesta múltiple, a su vez, para averiguar la asociación entre dos variables se ha utilizado el test Chi-cuadrado de Pearson ${ }^{12}$.

\section{Resultados}

\subsection{Utilización del cuerpo y su desvinculación con el producto promocionado}

Al tener en cuenta los personajes desvinculados del bien anunciado cabe destacar que no se producen diferencias estadísticamente significativas ni por sexo ni por año del festival, ya que los spots no suelen recurrir a este tipo de representación. Pese a la escasa utilización de esta técnica, se puede señalar que los manifiestos publicitarios muestran a personajes masculinos sin vinculación con los productos promocionados en los dos años examinados (4 anuncios en 1999 y 3 en 2009) $)^{13}$, pero únicamente se recurre a los personajes femeninos en el año 1999 (2 spots) $)^{14}$ (ver tabla 1).

12 Cuando la significación asintótica (bilateral) es menor que el nivel alfa establecido $(0,05)$ habitualmente se considera el criterio de significación $\mathrm{p}<0,05$, que supone un nivel de confianza del $95 \%$ (Pita y Pértega, 2004)-, y el valor de Chi-cuadrado de Pearson es superior a 3,84 (según la distribución de Chi-cuadrado para 1 grado de libertad y un valor de alfa de 0,05 , ya que para conocer las diferencias por género y por año del festival se han empleado tablas de $2 \times 2$ ), se estima que se produce una relación estadísticamente significativa entre las variables examinadas (Pita y Pértega, 2004). Así, se admite la hipótesis alternativa $\left(\mathrm{H}_{\mathrm{a}}=\right.$ sí existe asociación entre variables $)$ y se descarta la hipótesis nula $\left(\mathrm{H}_{0}=\right.$ las variables son independientes) (Pita y Pértega, 2004). No obstante, al haber realizado tablas de 2x2 hay que tener en cuenta que cuando más del $20 \%$ de las casillas tienen una frecuencia esperada inferior a cinco se recurre al estadístico exacto de Fisher (Pita y Pértega, 2004), en el que no se puede tener en cuenta el valor de la prueba, pero sí el de p.

${ }^{13}$ En el año 1999 estos spots fueron creados por las agencias Impoolsion, FCB / Tapsa, Tandem Campmany Guasch DDB y Young \& Rubicam para los anunciantes Vichy Catalán, Flex, Audi y Cachemir respectivamente. En 2009 estos manifiestos publicitarios promocionaban las pizzas Carrefour, la marca Vodafone y los supermercados Gadis, así, fueron elaborados por Publicis Comunicación España, Tapsa Madrid y Bap \& Conde.

141 anuncio de Canal 9 realizado por la agencia A Uno Asociados Estrategias y 1 spot de Audi elaborado por Tandem Campmany Guasch DDB. 
Tabla 1: Utilización del cuerpo masculino y femenino desvinculado del producto promocionado por año

\begin{tabular}{|c|c|c|c|c|}
\hline Cuerpos desvinculados ${ }^{\mathrm{a}}$ & Anuncios & 1999 & 2009 & TOTAL \\
\hline \multirow{4}{*}{ Masculinos } & Recuento & 4 & 3 & 7 \\
\hline & \% cuerpo & 57,1 & 42,9 & \\
\hline & $\%$ año & 66,7 & 100 & \\
\hline & $\%$ total & 44,4 & 33,3 & 77,8 \\
\hline \multirow{4}{*}{ Femeninos } & Recuento & 2 & 0 & 2 \\
\hline & $\%$ cuerpo & 100 & 0 & \\
\hline & $\%$ año & 33,3 & 0 & \\
\hline & $\%$ total & 22,2 & 0 & 22,2 \\
\hline \multirow{2}{*}{ TOTAL } & Recuento & 6 & 3 & 9 \\
\hline & $\%$ total & 66,7 & 33,3 & 100 \\
\hline
\end{tabular}

Fuente: elaboración propia ${ }^{15}$

Con referencia al grado de desnudez de los personajes, cabe destacar que en los dos niveles considerados se producen diferencias estadísticamente significativas por sexo, de esta forma, los hombres aparecen con mayor frecuencia vestidos (47,5\% del total frente a 40,6\%) $\left(\mathrm{x}^{2}=17,201 ; \mathrm{p}=0,000\right)$ y las mujeres se muestran más asiduamente semidesnudas o desnudas $(7 \%$ vs. $4,9 \%)(\mathrm{p}=0,001)$. Por su parte, no se producen diferencias estadísticamente significativas en función del año del festival, pese a estos datos, el número de anuncios que muestran a hombres y a mujeres no vestidos totalmente aumenta ligeramente en 2009 (ver tabla 2).

Tabla 2: Grado de desnudez para hombres y mujeres por año

\begin{tabular}{|c|c|c|c|c|}
\hline Grado de desnudez ${ }^{\mathrm{a}}$ & Anuncios & 1999 & 2009 & TOTAL \\
\hline Hombres vestidos & $\begin{array}{c}\text { Recuento } \\
\% \text { grado de desnudez } \\
\% \text { año } \\
\% \text { total } \\
\end{array}$ & $\begin{array}{c}60 \\
51,7 \\
49,2 \\
24,6 \\
\end{array}$ & $\begin{array}{c}56 \\
48,3 \\
45,9 \\
23 \\
\end{array}$ & 116 \\
\hline $\begin{array}{l}\text { Hombres no vestidos totalmente } \\
\text { (desnudos o semidesnudos) }\end{array}$ & $\begin{array}{c}\text { Recuento } \\
\% \text { grado de desnudez } \\
\% \text { año } \\
\% \text { total } \\
\end{array}$ & $\begin{array}{c}5 \\
41,7 \\
4,1 \\
2 \\
\end{array}$ & $\begin{array}{c}7 \\
58,3 \\
5,7 \\
2,9 \\
\end{array}$ & 4,9 \\
\hline Mujeres vestidas & $\begin{array}{c}\text { Recuento } \\
\% \text { grado de desnudez } \\
\% \text { año } \\
\% \text { total }\end{array}$ & $\begin{array}{c}49 \\
49,5 \\
40,2 \\
20,1\end{array}$ & $\begin{array}{c}50 \\
50,5 \\
41 \\
20,5\end{array}$ & 40,6 \\
\hline $\begin{array}{l}\text { Mujeres no vestidas totalmente } \\
\text { (desnudas o semidesnudas) }\end{array}$ & $\begin{array}{c}\text { Recuento } \\
\% \text { grado de desnudez } \\
\% \text { año } \\
\% \text { total }\end{array}$ & $\begin{array}{c}8 \\
47,1 \\
6,6 \\
3,3\end{array}$ & $\begin{array}{c}9 \\
52,9 \\
7,4 \\
3,7\end{array}$ & 17 \\
\hline TOTAL & $\begin{array}{c}\text { Recuento } \\
\% \text { total }\end{array}$ & $\begin{array}{c}122 \\
50\end{array}$ & $\begin{array}{c}122 \\
50\end{array}$ & $\begin{array}{l}244 \\
100\end{array}$ \\
\hline
\end{tabular}

Fuente: elaboración propia ${ }^{16}$

${ }^{15}$ Los porcentajes y los totales se basan en las respuestas. ${ }^{\text {a }}$ Agrupación de dicotomías. Tabulado el valor 1. 


\subsection{Presentación estereotípica de mujeres y hombres}

\subsubsection{Localizaciones}

Como se puede observar en la tabla 3, el hombre aparece con mayor asiduidad que la mujer en la esfera pública $(22,3 \%$ del total frente a $18,4 \%)\left(x^{2}=57,385 ; p=0,000\right)$. Sin embargo, tanto varones como féminas son mostrados con mayor frecuencia en el ámbito público que en el privado, sin que suela existir relación entre ambos ámbitos, es decir, cuando los personajes se ubican en el espacio público mayoritariamente no lo hacen como una extensión de su papel en el espacio privado.

No obstante, se producen diferencias estadísticamente significativas por sexo, de forma que es más habitual encontrar a los varones en la esfera pública sin relación con la privada $(21,4 \%$ frente a $18 \%)\left(x^{2}=57,816 ; p=0,000\right)$ y a las mujeres en el ámbito público con relación con el privado $(2,1 \%$ vs. $1,5 \%)(p=0,000)$.

Al tener en cuenta el año del festival, cabe resaltar que únicamente se producen diferencias estadísticamente significativas en el caso de mostrar a los personajes en la esfera pública sin relación con la privada. De esta forma, el porcentaje de mujeres y hombres que aparecen en esta situación es ligeramente superior en 2009.

Tabla 3: Localización de los personajes masculinos y femeninos por año

\begin{tabular}{|c|c|c|c|c|}
\hline Localización $^{\mathrm{a}}$ & Anuncios & 1999 & 2009 & TOTAL \\
\hline \multirow{4}{*}{ Hombres en la esfera pública } & Recuento & 51 & 53 & 104 \\
\hline & \% localización & 49 & 51 & \\
\hline & $\%$ año & 21,7 & 22,8 & \\
\hline & $\%$ total & 10,9 & 11,3 & 22,3 \\
\hline \multirow{4}{*}{ Hombres en la esfera privada } & Recuento & 19 & 19 & 38 \\
\hline & \% localización & 50 & 50 & \\
\hline & $\%$ año & 8,1 & 8,2 & \\
\hline & $\%$ total & 4,1 & 4,1 & 8,1 \\
\hline \multirow{4}{*}{$\begin{array}{l}\text { Hombres en la esfera pública sin } \\
\text { relación con la privada }\end{array}$} & Recuento & 48 & 52 & 100 \\
\hline & \% localización & 48 & 52 & \\
\hline & $\%$ año & 20,4 & 22,4 & \\
\hline & $\%$ total & 10,3 & 11,1 & 21,4 \\
\hline \multirow{4}{*}{$\begin{array}{c}\text { Hombres en la esfera pública como } \\
\text { extensión de la privada }\end{array}$} & Recuento & 5 & 2 & 7 \\
\hline & \% localización & 71,4 & 28,6 & \\
\hline & $\%$ año & 2,1 & 0,9 & \\
\hline & $\%$ total & 1,1 & 0,4 & 1,5 \\
\hline
\end{tabular}

${ }^{16}$ Los porcentajes y los totales se basan en las respuestas. ${ }^{a}$ Agrupación de dicotomías. Tabulado el valor 1. 


\begin{tabular}{|c|c|c|c|c|}
\hline Localización & Anuncios & 1999 & 2009 & TOTAL \\
\hline Mujeres en la esfera pública & $\begin{array}{c}\text { Recuento } \\
\% \text { localización } \\
\% \text { año } \\
\% \text { total }\end{array}$ & $\begin{array}{c}43 \\
50 \\
18,3 \\
9,2\end{array}$ & $\begin{array}{c}43 \\
50 \\
18,5 \\
9,2\end{array}$ & 86 \\
\hline Mujeres en la esfera privada & $\begin{array}{c}\text { Recuento } \\
\% \text { localización } \\
\% \text { año } \\
\% \text { total }\end{array}$ & $\begin{array}{c}20 \\
52,6 \\
8,5 \\
4,3\end{array}$ & $\begin{array}{c}18 \\
47,4 \\
7,8 \\
3,9\end{array}$ & 8,1 \\
\hline $\begin{array}{l}\text { Mujeres en la esfera pública sin } \\
\text { relación con la privada }\end{array}$ & $\begin{array}{c}\text { Recuento } \\
\% \text { localización } \\
\% \text { año } \\
\% \text { total }\end{array}$ & $\begin{array}{c}41 \\
48,8 \\
17,4 \\
8,8\end{array}$ & $\begin{array}{c}43 \\
51,2 \\
18,5 \\
9,2\end{array}$ & 18 \\
\hline $\begin{array}{l}\text { Mujeres en la esfera pública como } \\
\text { extensión de la privada }\end{array}$ & $\begin{array}{c}\text { Recuento } \\
\% \text { localización } \\
\% \text { año } \\
\% \text { total }\end{array}$ & $\begin{array}{c}8 \\
80 \\
3,4 \\
1,7\end{array}$ & $\begin{array}{c}2 \\
20 \\
0,9 \\
0,4\end{array}$ & 2,1 \\
\hline TOTAL & $\begin{array}{l}\text { Recuento } \\
\% \text { total }\end{array}$ & $\begin{array}{l}235 \\
50,3\end{array}$ & $\begin{array}{l}232 \\
49,7\end{array}$ & $\begin{array}{l}467 \\
100\end{array}$ \\
\hline
\end{tabular}

Fuente: elaboración propia ${ }^{17}$

\subsubsection{Profesiones}

Es más habitual encontrar a los personajes masculinos realizando alguna tarea remunerada que a los femeninos, pues los varones aparecen en esta situación en 93 spots y las féminas únicamente en 43.

Al tener en cuenta el sexo asociado tradicionalmente a las profesiones desempeñadas por los personajes de los anuncios analizados, cabe señalar que se producen diferencias estadísticamente significativas por sexo en el caso de los empleos considerados femeninos, de forma que estas tareas son desarrollas con mayor frecuencia por mujeres $(6,6 \%$ del total frente a $2,9 \%)(p=0,001)$.

Por su parte, las profesiones neutras también poseen este tipo de disimilitudes, no obstante, en esta ocasión los anuncios muestran que estos trabajos son realizadas mayoritariamente por hombres $(27,2 \%$ vs. $21,3 \%)\left(x^{2}=37,574 ; p=0,000\right)$. Pese a no existir diferencias estadísticamente significativas, también son los varones los que poseen con mayor asiduidad empleos masculinos (38,2\% frente a 3,7\%) (ver tabla 4$)$.

Por su parte, no existen diferencias estadísticamente significativas por año del festival. Sin embargo, se puede afirmar que en 2009 es más frecuente encontrar anuncios que muestran a hombres y a mujeres desempeñando tareas remuneradas, excepto en el caso de la población femenina y las profesiones asociadas tradicionalmente a los varones, ya que esta situación es más habitual en el año 1999.

${ }^{17}$ Los porcentajes y los totales se basan en las respuestas. ${ }^{a}$ Agrupación de dicotomías. Tabulado el valor 1. 
Tabla 4: Profesiones de los personajes masculinos y femeninos por año

\begin{tabular}{|c|c|c|c|c|}
\hline Profesiones $^{\mathrm{a}}$ & Anuncios & 1999 & 2009 & TOTAL \\
\hline \multirow{4}{*}{$\begin{array}{l}\text { Hombres con profesiones } \\
\text { típicamente masculinas }\end{array}$} & Recuento & 23 & 29 & 52 \\
\hline & $\%$ profesiones & 44,2 & 55,8 & \\
\hline & $\%$ año & 39 & 37,7 & \\
\hline & $\%$ total & 16,9 & 21,3 & 38,2 \\
\hline \multirow{4}{*}{$\begin{array}{l}\text { Hombres con profesiones } \\
\text { típicamente femeninas }\end{array}$} & Recuento & 1 & 3 & 4 \\
\hline & $\%$ profesiones & 25 & 75 & \\
\hline & $\%$ año & 1,7 & 3,9 & \\
\hline & $\%$ total & 0,7 & 2,2 & 2,9 \\
\hline \multirow{4}{*}{$\begin{array}{c}\text { Hombres con profesiones } \\
\text { neutras }\end{array}$} & Recuento & 17 & 20 & 37 \\
\hline & $\%$ profesiones & 45,9 & 54,1 & \\
\hline & \% año & 28,8 & 26 & \\
\hline & $\%$ total & 12,5 & 14,7 & 27,2 \\
\hline \multirow{4}{*}{$\begin{array}{l}\text { Mujeres con profesiones } \\
\text { típicamente masculinas }\end{array}$} & Recuento & 4 & 1 & 5 \\
\hline & $\%$ profesiones & 80 & 20 & \\
\hline & $\%$ año & 6,8 & 1,3 & \\
\hline & $\%$ total & 2,9 & 0,7 & 3,7 \\
\hline \multirow{4}{*}{$\begin{array}{l}\text { Mujeres con profesiones } \\
\text { típicamente femeninas }\end{array}$} & Recuento & 3 & 6 & 9 \\
\hline & $\%$ profesiones & 33,3 & 66,7 & \\
\hline & $\%$ año & 5,1 & 7,8 & \\
\hline & $\%$ total & 2,2 & 4,4 & 6,6 \\
\hline \multirow{4}{*}{$\begin{array}{l}\text { Mujeres con profesiones } \\
\text { neutras }\end{array}$} & Recuento & 11 & 18 & 29 \\
\hline & $\%$ profesiones & 37,9 & 62,1 & \\
\hline & $\%$ año & 18,6 & 23,4 & \\
\hline & $\%$ total & 8,1 & 13,2 & 21,3 \\
\hline \multirow{2}{*}{ TOTAL } & Recuento & 59 & 77 & 136 \\
\hline & $\%$ total & 43,4 & 56,7 & 100 \\
\hline
\end{tabular}

Fuente: elaboración propia $^{18}$

\subsubsection{Roles}

La tabla 5 pone de manifiesto que los tres roles más frecuentes desempeñados por hombres y mujeres coinciden, no obstante, el orden de aparición varía en función del sexo. Así, para los varones el papel más habitual es el de trabajador de la esfera pública (22,4\% del total), seguido del de usuario $(20,3 \%)$ y del de padre $(5,2 \%)$, aunque éste último se sitúa a gran distancia del anterior. En el caso de las mujeres, el rol más frecuente es el de usuaria $(15,4 \%)$, a continuación se localizan el de trabajadora de la esfera pública $(10,8)$ y el de madre $(6,2 \%)$.

Al tener en cuenta las diferencias por sexo, cabe destacar que estas son estadísticamente significativas en siete de los ocho roles considerados. De esta forma, la mujer únicamente aparece con mayor frecuencia que el hombre cuando es representada como madre $(6,2 \%$ del total vs. $5,2 \%)(p=0,000)$, por tanto, el hombre se muestra con mayor asiduidad que la mujer

${ }^{18}$ Los porcentajes y los totales se basan en las respuestas. ${ }^{\text {a }}$ Agrupación de dicotomías. Tabulado el valor 1. 
cuando es trabajador de la esfera pública $(22,4 \%$ frente a $10,8 \%)\left(x^{2}=7,511 ; \mathrm{p}=0,006\right)$, usuario $(20,3 \%$ frente a $15,4 \%)\left(x^{2}=26,911 ; p=0,000\right)$, objeto sexual $(3,4 \%$ vs. $2,8 \%)(p=0,000)$, seductor $(1,8 \%$ frente a $1,2 \%)(p=0,000)$, dependiente $(2,8 \%$ vs. $0,6 \%) \quad(p=0,003)$ e independiente $(2,5 \%$ vs. $1,8 \%)(\mathrm{p}=0,034)$.

Por su parte, la única representación que no muestra diferencias estadísticamente significativas por sexo es la de persona preocupada por su aspecto físico y por su vestuario, pues hombres y mujeres aparecen desempeñando este papel en el mismo porcentaje de anuncios $(1,5 \%)$.

Pese a estos datos, cabe señalar que solamente se producen disimilitudes estadísticamente significativas por año del festival en este rol para los personajes femeninos $(\mathrm{p}=0,020)$, ya que no se ha podido encontrar esta representación de la mujer en ningún anuncio de 1999, pero sí aparece en 5 de 2009.

Tabla 5: Roles masculinos y femeninos por año

\begin{tabular}{|c|c|c|c|c|}
\hline Papel $^{\mathbf{a}}$ & Anuncios & 1999 & 2009 & TOTAL \\
\hline Hombre objeto sexual & $\begin{array}{c}\text { Recuento } \\
\% \text { estereotipos } \\
\% \text { año } \\
\% \text { total }\end{array}$ & $\begin{array}{c}5 \\
45,5 \\
3,1 \\
1,5\end{array}$ & $\begin{array}{c}6 \\
54,5 \\
3,6 \\
1,8\end{array}$ & 11 \\
\hline Hombre usuario & $\begin{array}{c}\text { Recuento } \\
\% \text { estereotipos } \\
\% \text { año } \\
\% \text { total }\end{array}$ & $\begin{array}{c}34 \\
51,5 \\
21,3 \\
10,5\end{array}$ & $\begin{array}{c}32 \\
48,5 \\
19,4 \\
9,8\end{array}$ & 20,3 \\
\hline $\begin{array}{c}\text { Hombre trabajador de la } \\
\text { esfera pública }\end{array}$ & $\begin{array}{c}\text { Recuento } \\
\% \text { estereotipos } \\
\% \text { año } \\
\% \text { total }\end{array}$ & $\begin{array}{c}35 \\
48,6 \\
21,9 \\
10,8\end{array}$ & $\begin{array}{c}37 \\
51,4 \\
22,4 \\
11,4\end{array}$ & 22,4 \\
\hline Hombre padre & $\begin{array}{c}\text { Recuento } \\
\% \text { estereotipos } \\
\% \text { año } \\
\% \text { total }\end{array}$ & $\begin{array}{c}6 \\
35,3 \\
3,8 \\
1,9\end{array}$ & $\begin{array}{c}11 \\
64,7 \\
6,7 \\
3,4\end{array}$ & 17 \\
\hline Hombre seductor & $\begin{array}{c}\text { Recuento } \\
\% \text { estereotipos } \\
\% \text { año } \\
\% \text { total }\end{array}$ & $\begin{array}{c}3 \\
50 \\
1,9 \\
0,9\end{array}$ & $\begin{array}{c}3 \\
50 \\
1,8 \\
0,9\end{array}$ & 1,8 \\
\hline $\begin{array}{l}\text { Hombre preocupado } \\
\text { aspecto físico y vestuario }\end{array}$ & $\begin{array}{c}\text { Recuento } \\
\% \text { estereotipos } \\
\% \text { año } \\
\% \text { total }\end{array}$ & $\begin{array}{c}1 \\
20 \\
0,6 \\
0,3\end{array}$ & $\begin{array}{c}4 \\
80 \\
2,4 \\
1,2\end{array}$ & 1,5 \\
\hline Hombre dependiente & $\begin{array}{c}\text { Recuento } \\
\% \text { estereotipos } \\
\% \text { año } \\
\% \text { total }\end{array}$ & $\begin{array}{c}4 \\
44,4 \\
2,5 \\
1,2\end{array}$ & $\begin{array}{c}5 \\
55,6 \\
3 \\
1,5\end{array}$ & 2,8 \\
\hline
\end{tabular}




\begin{tabular}{|c|c|c|c|c|}
\hline Papel & Anuncios & 1999 & 2009 & TOTAL \\
\hline \multirow{4}{*}{ Hombre independiente } & Recuento & 6 & 2 & 8 \\
\hline & $\%$ estereotipos & 75 & 25 & \\
\hline & $\%$ año & 3,8 & 1,2 & \\
\hline & $\%$ total & 1,8 & 0,6 & 2,5 \\
\hline \multirow{4}{*}{ Mujer objeto sexual } & Recuento & 5 & 4 & 9 \\
\hline & $\%$ estereotipos & 55,6 & 44,4 & \\
\hline & $\%$ año & 3,1 & 2,4 & \\
\hline & $\%$ total & 1,5 & 1,2 & 2,8 \\
\hline \multirow{4}{*}{ Mujer usuaria } & Recuento & 29 & 21 & 50 \\
\hline & $\%$ estereotipos & 58 & 42 & \\
\hline & $\%$ año & 18,1 & 12,7 & \\
\hline & $\%$ total & 8,9 & 6,5 & 15,4 \\
\hline \multirow{4}{*}{$\begin{array}{l}\text { Mujer trabajadora de la } \\
\text { esfera pública }\end{array}$} & Recuento & 16 & 19 & 35 \\
\hline & $\%$ estereotipos & 45,7 & 54,3 & \\
\hline & $\%$ año & 10 & 11,5 & \\
\hline & $\%$ total & 4,9 & 5,8 & 10,8 \\
\hline \multirow{4}{*}{ Mujer madre } & Recuento & 10 & 10 & 20 \\
\hline & $\%$ estereotipos & 50 & 50 & \\
\hline & $\%$ año & 6,3 & 6,1 & \\
\hline & $\%$ total & 3,1 & 3,1 & 6,2 \\
\hline \multirow{4}{*}{ Mujer seductora } & Recuento & 2 & 2 & 4 \\
\hline & $\%$ estereotipos & 50 & 50 & \\
\hline & $\%$ año & 1,3 & 1,2 & \\
\hline & $\%$ total & 0,6 & 0,6 & 1,2 \\
\hline \multirow{4}{*}{$\begin{array}{l}\text { Mujer preocupada aspecto } \\
\text { físico y vestuario }\end{array}$} & Recuento & 0 & 5 & 5 \\
\hline & $\%$ estereotipos & 0 & 100 & \\
\hline & $\%$ año & 0 & 3 & \\
\hline & $\%$ total & 0 & 1,5 & 1,5 \\
\hline \multirow{4}{*}{ Mujer dependiente } & Recuento & 0 & 2 & 2 \\
\hline & $\%$ estereotipos & 0 & 100 & \\
\hline & $\%$ año & 0 & 1,2 & \\
\hline & $\%$ total & 0 & 0,6 & 0,6 \\
\hline \multirow{4}{*}{ Mujer independiente } & Recuento & 4 & 2 & 6 \\
\hline & $\%$ estereotipos & 66,7 & 33,3 & \\
\hline & $\%$ año & 2,5 & 1,2 & \\
\hline & $\%$ total & 1,2 & 0,6 & 1,8 \\
\hline \multirow{2}{*}{ TOTAL } & Recuento & 160 & 165 & 325 \\
\hline & $\%$ total & 49,2 & 50,8 & 100 \\
\hline
\end{tabular}

Fuente: elaboración propia ${ }^{19}$

\subsubsection{Argumentos de persuasión}

Pese a no existir diferencias estadísticamente significativas por sexo ni por año del festival con relación a los argumentos de persuasión dados por los personajes, cabe destacar

\footnotetext{
${ }^{19}$ Los porcentajes y los totales se basan en las respuestas. ${ }^{a}$ Agrupación de dicotomías. Tabulado el valor 1.
} 
que el hombre aparece con mayor asiduidad que la mujer ofreciendo premisas, ya sean éstas racionales o emocionales.

Especialmente llamativa es la disimilitud existente en la argumentación racional, pues alcanza los 34,3 puntos del total. Por su parte, las desemejanzas encontradas en los años analizados no son llamativas, sin embargo, se puede señalar que las mujeres aparecen en un mayor número de anuncios ofreciendo argumentos, ya sean éstos emocionales o racionales, en 2009 (ver tabla 6).

Tabla 6: Argumentos dados por los personajes masculinos y femeninos por año

\begin{tabular}{|c|c|c|c|c|}
\hline Argumentos $^{\mathbf{a}}$ & Anuncios & $\mathbf{1 9 9 9}$ & $\mathbf{2 0 0 9}$ & TOTAL \\
\hline \multirow{3}{*}{ Hombres que dan } & Recuento & 44 & 42 & 86 \\
argumentos racionales & \% argumentos & 51,2 & 48,8 & \\
& \% año & 51,2 & 45,7 & \\
& \% total & 24,7 & 23,6 & 48,3 \\
\hline \multirow{3}{*}{ Hombres que dan } & Recuento & 27 & 27 & 54 \\
argumentos emocionales & \% argumentos & 50 & 50 & \\
& \% año & 31,4 & 29,3 & \\
& \% total & 15,2 & 15,2 & 30,3 \\
\hline \multirow{3}{*}{ Mujeres que dan } & Recuento & 11 & 14 & 25 \\
argumentos racionales & \% argumentos & 44 & 56 & \\
& \% año & 12,8 & 15,2 & \\
& \% total & 6,2 & 7,9 & 14 \\
\hline \multirow{2}{*}{ Mujeres que dan } & Recuento & 4 & 9 & 13 \\
argumentos emocionales & \% argumentos & 30,8 & 69,2 & \\
& \% año & 4,7 & 9,8 & \\
& \% total & 2,2 & 5,1 & 7,3 \\
\hline \multirow{2}{*}{ TOTAL } & Recuento & 86 & 92 & 178 \\
& \% total & 48,3 & 51,7 & 100 \\
\hline
\end{tabular}

Fuente: elaboración propia ${ }^{20}$

\subsection{Sesgos de género}

\subsubsection{Visibilidad}

Se producen diferencias estadísticamente significativas por sexo tanto en la aparición de personajes en el video $\left(x^{2}=8,739 ; p=0,003\right)$ como en el audio $\left(x^{2}=9,249 ; p=0,002\right)$, de forma que la visibilidad de los varones es superior a la de las féminas en las dos ocasiones consideradas. En concreto, se pueden observar hombres de forma visual en el 32,8\% del total

${ }^{20}$ Los porcentajes y los totales se basan en las respuestas. ${ }^{a}$ Agrupación de dicotomías. Tabulado el valor 1. 
de los anuncios analizados frente al $29,3 \%$ en el que se muestran mujeres, aunque especialmente llamativa es la distancia existen en las voces en off, pues alcanza los 21,1 puntos (ver tabla 7).

Por su parte, no existen disimilitudes estadísticamente significativas en función del año del festival entre la visibilidad masculina y la femenina. Pese a esta información, es más frecuente encontrar anuncios que muestran a hombres en 1999 que en 2009, ya sea de forma visual (64 spots frente a 57) o sonora (63 vs. 46). En el caso de las féminas, éstas aparecen representadas de forma visual con mayor asiduidad en la publicidad de 1999 (57 anuncios frente a 51), sin embargo, el número de manifiestos publicitarios que utilizan voces en off femeninas es mayor en 2009 (14 vs.17).

Tabla 7: Visibilidad de los personajes masculinos y femeninos por año

\begin{tabular}{|c|c|c|c|c|}
\hline Visibilidad & Anuncios & $\mathbf{1 9 9 9}$ & $\mathbf{2 0 0 9}$ & TOTAL \\
\hline \multirow{4}{*}{ Hombres que aparecen de } & Recuento & 64 & 57 & 121 \\
forma visual & \% visibilidad & 52,9 & 47,1 & \\
& \% año & 32,3 & 33,3 & \\
& \% total & 17,3 & 15,4 & 32,8 \\
\hline \multirow{3}{*}{ Voces en off masculinas } & Recuento & 63 & 46 & 109 \\
& \% visibilidad & 57,8 & 42,2 & \\
& \% año & 31,8 & 26,9 & \\
Mujeres que aparecen de & \% total & 17,1 & 12,5 & 29,5 \\
\hline forma visual & Recuento & 57 & 51 & 108 \\
& \% visibilidad & 52,8 & 47,2 & \\
& \% año & 28,8 & 29,8 & \\
& Recuento & 15,4 & 13,8 & 29,3 \\
\hline \multirow{3}{*}{ Voces en off femeninas } & \% visibilidad & 45,2 & 54,8 & 31 \\
& \% año & 7,1 & 9,9 & \\
& \% total & 3,8 & 4,6 & 8,4 \\
\hline \multirow{2}{*}{ TOTAL } & Recuento & 198 & 171 & 369 \\
& \% total & 53,7 & 46,3 & 100 \\
\hline
\end{tabular}

Fuente: elaboración propia ${ }^{21}$

\subsubsection{Paridad}

Con referencia al sexo se pueden observar diferencias estadísticamente significativas en el caso de los personajes que hacen $\left(x^{2}=9,576 ; p=0,002\right)$ y que padecen $\left(x^{2}=10,373 ; p=0,001\right)$. Así, los hombres hacen en mayor medida que las mujeres $(25,7 \%$ del total frente a $22,3 \%)$ y son receptores de acciones de forma más habitual que los féminas $(9,1 \%$ del total vs. 3,8\%).

${ }^{21}$ Los porcentajes y los totales se basan en las respuestas. ${ }^{\text {a }}$ Agrupación de dicotomías. Tabulado el valor 1. 
Pese a no haber disimilitudes estadísticamente significativas, los personajes masculinos también dicen con mayor asiduidad que los femeninos (27\% vs. 12,1\%) (ver tabla 8 ).

$\mathrm{Al}$ igual que en el caso anterior, no se producen diferencias estadísticamente significativas en función del año del festival, de esta forma, las mujeres dicen, hacen y padecen en un número de anuncios muy similar, o incluso idéntico. No obstante, en el caso de los varones se producen mayores desigualdades por año del festival, especialmente destacable es la distancia existente en los varones que dicen, pues alcanza los 15 spots (71 anuncios frente 56 ).

Tabla 8: Paridad de los personajes masculinos y femeninos por año

\begin{tabular}{|c|c|c|c|c|}
\hline Paridad $^{\text {a }}$ & Anuncios & $\mathbf{1 9 9 9}$ & $\mathbf{2 0 0 9}$ & TOTAL \\
\hline \multirow{4}{*}{ Hombres dicen } & Recuento & 71 & 56 & 127 \\
& \% paridad & 55,9 & 44,1 & \\
& \% año & 28,6 & 25,1 & \\
& \% total & 15,1 & 11,9 & 27 \\
\hline \multirow{4}{*}{ Hombres hacen } & Recuento & 64 & 57 & 121 \\
& \% paridad & 52,9 & 47,1 & \\
& \% año & 25,8 & 25,6 & \\
& \% total & 13,6 & 12,1 & 25,7 \\
\hline \multirow{5}{*}{ Hombres padecen } & Recuento & 19 & 24 & 43 \\
& \% paridad & 44,2 & 55,8 & \\
& \% año & 7,7 & 10,8 & \\
& \% total & 4 & 5,1 & 9,1 \\
\hline \multirow{5}{*}{ Mujeres dicen } & Recuento & 30 & 27 & 57 \\
& \% paridad & 52,6 & 47,4 & \\
& \% año & 12,1 & 12,1 & \\
& \% total & 6,4 & 5,7 & 12,1 \\
\hline \multirow{3}{*}{ Mujeres hacen } & Recuento & 55 & 50 & 105 \\
& \% paridad & 52,4 & 47,6 & \\
& \% año & 22,2 & 22,4 & \\
& \% total & 11,7 & 10,6 & 22,3 \\
\hline \multirow{3}{*}{ Mujeres padecen } & Recuento & 9 & 9 & 18 \\
& \% paridad & 50 & 50 & \\
& \% año & 3,6 & 4 & \\
& \% total & 1,9 & 1,9 & 3,8 \\
\hline \multirow{2}{*}{ TOTAL } & Recuento & 248 & 223 & 471 \\
& \% total & 52,7 & 47,3 & 100 \\
\hline
\end{tabular}

Fuente: elaboración propia ${ }^{22}$

\subsubsection{Empoderamiento}

Al tener en cuenta el sexo, cabe destacar que se observan diferencias estadísticamente significativas, favorables hacia los varones, cuando se muestran personajes tomando decisiones, en concreto, en 35 anuncios aparecen hombres en esta situación frente a los 16 spots que utilizan a mujeres. Pese a no producirse diferencias estadísticamente significativas,

${ }^{22}$ Los porcentajes y los totales se basan en las respuestas. ${ }^{a}$ Agrupación de dicotomías. Tabulado el valor 1. 
los varones son presentados haciendo aquello que se les recomienda en 11 anuncios frente a los 2 en los que se representa a mujeres en esta situación (ver tabla 9).

En esta ocasión, tampoco se producen diferencias estadísticamente significativas en función del año del festival. Pese a esta información, se puede señalar que tanto hombres como mujeres aparecen en anuncios distintos tomando decisiones de forma más habitual en 2009, por su parte, los varones hacen aquello que se les recomienda de manera más frecuente en 1999 y las mujeres únicamente acatan órdenes en 1 anuncio de cada año considerado.

Tabla 9: Empoderamiento de los personajes masculinos y femeninos por año

\begin{tabular}{|c|c|c|c|c|}
\hline Empoderamiento $^{\mathbf{a}}$ & Anuncios & $\mathbf{1 9 9 9}$ & $\mathbf{2 0 0 9}$ & TOTAL \\
\hline \multirow{3}{*}{ Hombres que toman } & Recuento & 17 & 18 & 35 \\
decisiones & \% empoderamiento & 48,6 & 51,4 & \\
& \% año & 50 & 60 & \\
& \% total & 26,6 & 28,1 & 54,7 \\
\hline \multirow{2}{*}{ Hombres que hacen } & Recuento & 9 & 2 & 11 \\
aquello que se les & \% empoderamiento & 81,8 & 18,2 & \\
recomienda & \% año & 26,5 & 6,7 & \\
& \% total & 14,1 & 3,1 & 17,2 \\
\hline \multirow{2}{*}{ Mujeres que toman } & \% empoderamiento & 43,8 & 56,3 & 16 \\
decisiones & \% año & 20,6 & 30 & \\
& \% total & 10,9 & 14,1 & 25 \\
\hline \multirow{2}{*}{ Mujeres que hacen } & Recuento & 1 & 1 & 2 \\
aquello que se les & \% empoderamiento & 50 & 50 & \\
recomienda & \% año & 2,9 & 3,3 & \\
& \% total & 1,6 & 1,6 & 3,1 \\
\hline \multirow{2}{*}{ TOTAL } & Recuento & 34 & 30 & 64 \\
& \% total & 53,1 & 46,9 & 100 \\
\hline
\end{tabular}

Fuente: elaboración propia ${ }^{23}$

\section{Conclusiones y discusión}

Como ponen de manifiesto los resultados del presente trabajo, no existen grandes diferencias entre los spots presentados al festival "El Sol" en 1999 y en 2009 con referencia a la trasmisión del sexismo publicitario. Por tanto, se puede afirmar que la promulgación de la Ley Orgánica de Medidas de Protección Integral contra la Violencia de Género no ha conllevado cambios significativos en la discriminación por sexo mostrada en los spots.

Pese a esta situación, tras la entrada en vigor de la normativa analizada se deja de utilizar el cuerpo de las mujeres desvinculado del bien promocionado, aunque el empleo de esta técnica ya era muy escaso con anterioridad a la creación de esta ley.

${ }^{23}$ Los porcentajes y los totales se basan en las respuestas. ${ }^{a}$ Agrupación de dicotomías. Tabulado el valor 1. 
De todos los aspectos analizados, únicamente se observan diferencias estadísticamente significativas por año en dos ocasiones. En una de ellas se establece que es más frecuente mostrar a hombres y a las mujeres en la esfera pública sin que esta localización tenga relación con el ámbito privado en la publicidad del año 2009. La otra situación tiene en cuenta los roles desempeñados por los personajes femeninos, en concreto, la representación de las mujeres preocupadas por su aspecto físico y por su vestuario, pues este papel solamente se puede observar tras la promulgación de la ley considerada, situación debida, quizás, a que en la publicidad cada vez más se muestra un modelo de mujer centrado en la sensualidad y en la exhibición y cuidado del cuerpo (Cáceres y Díaz, 2008).

No obstante, se puede observar una amplia gama de diferencias significativas en función del sexo. Así, pese a que los personajes masculinos y femeninos son mostrados principalmente con ropa (García, 2003), cabe destacar que es más habitual encontrar a hombres vestidos que a mujeres y a féminas semidesnudas o desnudas que a varones (Reichert y Carpenter, 2004).

A su vez, al tener en cuenta la ubicación de los personajes es importante señalar que es más frecuente que en el ámbito público aparezcan los hombres (Bretl y Cantor, 1988; Uray y Burnaz, 2003). También es más habitual que los varones ostenten con mayor asiduidad el papel de trabajador de la esfera pública, pues en los manifiestos publicitarios los personajes masculinos suelen realizar con mayor asiduidad que los femeninos actividades laborales (Coltrane y Adams, 1997; Valls-Fernández y Martínez-Vicente, 2007). En este sentido, cabe señalar que el único rol en el que aparecen las mujeres de forma más habitual que los hombres es en el de madre, ya que la publicidad continúa representado a la población femenina en este papel (Berganza y Hoyo, 2006).

En el caso de los sesgos de género se observan diferencias estadísticamente significativas en función del sexo para los tres supuestos: visibilidad, paridad y empoderamiento. De esta forma, los hombres aparecen en los spots analizados con mayor frecuencia que las mujeres, tanto de forma visual como sonora (Berganza y Hoyo, 2006). Al tener en cuenta la paridad son los varones los que vuelven a adquirir protagonismo al ser el sexo que aparece en mayor número de anuncios haciendo y padeciendo acciones, situación debida, tal vez, a su amplia visibilidad. Con referencia al empoderamiento es importante señalar que son los personajes masculinos los que toman decisiones de forma más habitual, pese a la existencia de trabajos que aluden al aumento de la capacidad decisoria de los personajes publicitarios femeninos con el paso de los años (Royo, Aldás, Küster y Vila, 2005).

Al igual que "numerosos estudios han demostrado que la publicidad es sexista" (Moral, 2000: 209), la presente investigación pone de manifiesto que los spots analizados recurren a la discriminación por sexo. Por tanto, se puede afirmar que la promulgación de la Ley Orgánica de Medidas de Protección Integral contra la Violencia de Género no ha sido eficaz, ya que los manifiestos publicitarios muestran el mismo grado de sexismo cinco años antes y cinco años después de la creación de esta normativa. 
Finalmente, se puede señalar como la principal limitación de este trabajo el haber utilizado únicamente anuncios presentados al festival "El Sol". Por tanto, podría ser que si se seleccionase una muestra distinta los resultados no coincidieran con los obtenidos en este análisis. De esta forma, se abre una posible línea de investigación, pues sería interesante comprobar si las conclusiones obtenidas en este texto se mantienen al escoger una muestra de spots no presentados a concursos de creatividad.

\section{BIBLIOGRAFÍA}

- Argentina (2009): "Ley 26.485 de Protección Integral Para Prevenir, Sancionar y Erradicar la Violencia Contra las Mujeres en los Ámbitos en que Desarrollen sus Relaciones Interpersonales”. En Boletín Oficial de la República Argentina, 14 de abril de 2009, nº 31632, pp. 1-38, [en línea] Disponible en: http://www.boletinoficial.gov.ar/Inicio/index.castle?s=1\&fea=14/04/2009 [20/02/2012].

- Berganza, Ma Rosa; Hoyo, Mercedes del (2006): "La mujer y el hombre en la publicidad televisiva: imágenes y estereotipos". En Zer, $\mathrm{n}^{\mathrm{o}}$ 21, pp. 161-175, [en línea] Disponible en: http://www.ehu.es/ojs/index.php/Zer/article/view/3730/3360 [20/02/2012].

- Bretl, Daniel J. y Cantor, Joanne (1988): "The portrayal of men and women in U.S. television commercials: a recent content analysis and trends over 15 years". En Sex roles, Vol. 18, n 9-10, pp. 595-609, [en línea] Disponible en: http://www.springerlink.com/content/r170378810566433/ fulltext.pdf [20/02/2012].

- Bustelo, María (2001): La evolución de las políticas públicas de igualdad de género de los gobiernos central y autonómicos en España: 1995-1999. Tesis Doctoral, Madrid: Universidad Complutense de Madrid.

- Cáceres, María Dolores y Díaz, Paloma (2008): "La representación del cuerpo de la mujer en la publicidad de revistas femeninas". En Estudios sobre el mensaje periodístico, Vol. 14, pp. 309-327, [en línea] Disponible en: http://revistas.ucm.es/index.php/ESMP/article/view/ESMP0808110309A/ 11915 [20/02/2012].

- Coltrane, Scott; Adams, Michele (1997): "Work-family imagery and gender stereotypes: television and the reproduction of difference". En Journal of vocational behavior, Vol. 50, n 2, pp. 323-347.

- Dietz, Tracy L. (1998): “An examination of violence and gender role portrayals in video games: implications for gender socialization and aggressive behaviour". En Sex roles, Vol. 38, no 5-6, pp. 425-442, [en línea] Disponible en: http://www.springerlink.com/content/r326135512365r40/ fulltext.pdf [20/02/2012].

- España (2009): “Ley 29/2009, de 30 de diciembre, por la que se Modifica el Régimen Legal de la Competencia Desleal y de la Publicidad Para la Mejora de la Protección de los Consumidores y Usuarios". En Boletín Oficial del Estado, 31 de diciembre de 2009, n 315, pp. 112039-112060, [en línea] Disponible en: http://www.boe.es/diario_boe/txt.php?id=BOE-A-2009-21162 [20/02/2012].

- España (2007): “Ley Orgánica 3/2007, de 22 de marzo, Para la Igualdad Efectiva de Mujeres y Hombres". En Boletín Oficial del Estado, 23 de marzo de 2007, n 71, pp. 12611-12645, [en línea] Disponible en: http://www.boe.es/aeboe/consultas/bases_datos/act.php?id=BOE-A-2007-6115 
[20/02/2012].

- España (2006): "Ley 39/2006, de 14 de diciembre, de Promoción de la Autonomía Personal y Atención a las Personas en Situación de Dependencia". En Boletín Oficial del Estado, 15 de diciembre de 2006, $\mathrm{n}^{\mathrm{o}}$ 299, pp. 44142-44156, [en línea] Disponible en: http://www.boe.es/aeboe/consultas/bases_datos/act.php?id=BOE-A-2006-21990 [20/02/2012].

- España (2004): "Ley Orgánica 1/2004, de 28 de diciembre, de Medidas de Protección Integral Contra la Violencia de Género". En Boletín Oficial del Estado, 29 de diciembre de 2004, n 313, pp. 42166-42197, [en línea] Disponible en: http://www.boe.es/aeboe/consultas/bases_datos/act.php?id= BOE-A-2004-21760 [20/02/2012].

- España (1999): "Ley 39/1999, de 5 de noviembre, Para Promover la Conciliación de la Vida Familiar y Laboral de las Personas Trabajadoras". En: Boletín Oficial del Estado, 6 de noviembre de 1999, $\mathrm{n}^{\circ}$ 266, pp. 38934-38942, [en línea] Disponible en: http://www.boe.es/aeboe/consultas/ bases_datos/act.php?id=BOE-A-1999-21568 [20/02/2012].

- España (1988): “Ley 34/1988, de 11 de noviembre, General de Publicidad”. En Boletín Oficial del Estado, 15 de noviembre de 1988, $\mathrm{n}^{\circ}$ 274, pp. 32464-32467, [en línea] Disponible en: http://www.boe.es/aeboe/consultas/bases_datos/act.php?id=BOE-A-1988-26156 [20/02/2012].

- García del Castillo, José Antonio; López-Sánchez, Carmen; Quiles, Ma del Carmen y García del Castillo-López, Álvaro (2009): "Descripción y análisis de la publicidad de alcohol en la revista Muy Interesante". En Revista latina de comunicación social, $\mathrm{n}^{\mathrm{o}}$ 64, pp. 461-468, [en línea] Disponible en: http://www.revistalatinacs.org/09/art/38_837_Elche/23Quiles.html [20/02/2012].

- García, Marta (2010): "La voz de las mujeres en la universidad”. En Revista de la asociación de sociología de la educación, Vol. 3, $\mathrm{n}^{\mathrm{o}}$ 3, pp. 357-368, [en línea] Disponible en: http://www.ase.es:81/navegacion/subido/numerosRase/0303/03_3_GARCIA.pdf [20/02/2012].

- García, Patricia (2003): Estereotipos de género en publicidad televisiva. Tesis doctoral, Málaga: Universidad de Málaga.

- González, Juan José (2007): "Estereotipos familiares en el cine y la publicidad". En Carthaginensia, Vol. 23, no 44, pp. 415-430.

- Guarro, Beatriu (2004): "La publicidad televisiva como instrumento para el análisis de estereotipos sexistas". En VIII congreso sociedad española de didáctica de la lengua y la literatura. La Habana: Sociedad española de didáctica de la lengua y la literatura, [en línea] Disponible en: http://sedll.org/es/congresos_actas_interior.php?cod=274 [20/02/2012].

- Instituto de la Mujer (n.d.): "Mujeres muertas por violencia de género a manos de su pareja o expareja", [en línea] Disponible en: http://www.inmujer.gob.es/ss/Satellite?c=Page\&cid=1264005678228 \&language $=$ cas_ES\&pagename $=$ InstitutoMujer\%2FPage\%2FIMUJ_Estadisticas [20/02/2012].

- Instituto Nacional de Estadística (n.d.): "Encuesta de población activa”, [en línea] Disponible en: http://www.ine.es/jaxi/menu.do?type $=$ pcaxis $\&$ path $=/$ t22 $/ \mathrm{e} 308 \_$mnu $\&$ file $=$ inebase $\& N=\& \mathrm{~L}=0$ [20/02/2012].

- Kervin, Denise (1990): "Advertising masculinity: the representation of males in esquire advertisements". En Journal of communication inquiry, vol. 14, $\mathrm{n}^{\mathrm{o}}$ 1, pp. 51-70.

- Marrugat, Jaume; Vila, Joan; Pavesi, Marco y Sanz, Ferran (1998): "Estimación del tamaño de la muestra en la investigación clínica y epidemiológica”. En Medicina clínica, Vol. 111, nº 7, pp. 267-276.

- Martín-Llaguno, Marta; Quiles, M. Del Carmen y López, Carmen (2004): “Los sistemas de autorregulación como mecanismos de control de la publicidad de tabaco: evaluación mediante 
análisis empírico". En Gaceta sanitaria, Vol. 18, no 5, pp. 366-373, [en línea] Disponible en: http://apps.elsevier.es/watermark/ctl_servlet?_f=10\&pident_articulo=13067394\&pident_usuario $=0$ \&pcontactid $=\&$ pident_revista $=138 \&$ ty $=87 \&$ accion $=$ L\&origen $=$ elsevier\&web $=w w w . e l s e v i e r . e s \& l a$ $\mathrm{n}=$ es\&fichero=138v18n05a13067394pdf001.pdf [20/02/2012].

- México (2007): "Ley General de Acceso a las Mujeres a una Vida Libre de Violencia". En Diario Oficial de la Federación, 1 de febrero de 2007, pp. 2-17, [en línea] Disponible en: http://dof.gob.mx/nota_detalle.php?codigo=4961209\&fecha=01/02/2007 [20/02/2012].

- Moral, Ma Esther del (2000): "Los nuevos modelos de mujer y de hombre a través de la publicidad". En: Comunicar, Vol. 7, n 14, pp. 208-217, [en línea] Disponible en: http://www.revistacomunicar.com/ index.php?contenido=detalles\&numero=14\&articulo=14-2000-27 [20/02/2012].

- Navarro-Beltrá, Marián y Martín-Llaguno, Marta (2010): "La publicidad sexista según las leyes integrales de violencia de género. Estudio empírico en España y Argentina”. En II congreso internacional latina de comunicación social. La Laguna: Sociedad latina de comunicación social, [en línea] Disponible en: http://www.revistalatinacs.org/10SLCS/propuestas.html [20/02/2012].

- Pita, Salvador; Pértega, Sonia (2004): “Asociación de variables cualitativas: el test exacto de Fisher y el test de McNemar". En Cadernos de Atención Primaria, Vol. 11, n 5, pp. 304-308, [en línea] Disponible en: http://www.agamfec.com/pdf/CADERNOS/VOL11/VOL11_5/14_Invest_ N11_5.pdf [00/02/2012].

- Reichert, Tom y Carpenter, Courtney (2004): “An update on sex in magazine advertising: 1983 to 2003". En Journalism and mass communication quarterly, Vol. 81, n 4, pp. 823-837.

- Royo, Marcelo; Aldás, Joaquín; Küster, Inés; Vila, Natalia (2005): "Roles de género y sexismo en la publicidad de las revistas españolas: un análisis de las tres últimas décadas del siglo XX". En Comunicación y sociedad, Vol. 18, $\mathrm{n}^{\mathrm{o}} 1$, pp. 113-52, [en línea] Disponible en: http://www.unav.es/fcom/comunicacionysociedad/es/resumen.php?art_id=69 [20/02/2012].

- Ruiz, María Teresa et al. (2004): "El enfoque de género en las noticias de salud". En Gaceta sanitaria, Vol. 18, $\mathrm{n}^{\circ}$ Supl. 2, pp. 65-74, [en línea] Disponible en: http://apps.elsevier.es/watermark/ ctl_servlet?_f=10\&pident_articulo $=13061996 \&$ pident_usuario $=0$ \&pcontactid $=$ \&pident_revista $=138$ $\& \overline{t y}=161 \&$ accion $=$ L\&origen $=$ elsevier\&web $=w w w . e l s e v i e r . e s \& l a n=e s \&$ fichero $=138 \mathrm{v} 1 \overline{8} \mathrm{nSupl} .2 \mathrm{a} 13$ 061996pdf001.pdf [20/02/2012].

- Sigma Dos (2006): III macroencuesta sobre la violencia contra las mujeres. Madrid: Instituto de la Mujer, [en línea] Disponible en: http://www.unece.org/fileadmin/DAM/stats/gender/vaw/ surveys/Spain/publication.pdf [20/02/2012].

- Spirek, Melissa M. y Glascock, Jack (1998): "Gender analysis of frightening film newspaper advertisements: a 50 year overview (1940-1990)". En Communication quarterly, Vol. 46, nº 1, pp. 100-109.

- Uray, Nimet y Burnaz, Sebnem (2003): “An analysis of the portrayal of gender roles in Turkish television advertisements". En Sex roles, Vol. 48, n ${ }^{\circ}$ 1-2, pp. 77-87, [en línea] Disponible en: http://www.springerlink.com/content/g716m4p5g15v7r29/fulltext.pdf [20/02/2012].

- Valls-Fernández, Federico y Martínez-Vicente, José Manuel (2007): “Gender stereotypes in Spanish television commercials". En Sex roles, Vol. 56, nº 9-10, pp. 691-699, [en línea] Disponible en: http://www.springerlink.com/content/q0177270k8806147/fulltext.pdf [20/02/2012].

- Wimmer, Roger D. y Dominick, Joseph R. (1996): La investigación científica de los medios de comunicación. Barcelona: Bosch Casa Editorial. 